\title{
What Is the Therapeutic Mechanism of the Probiotics in Irritable Bowel Syndrome Patients With Visceral Hypersensitivity?
} (Neurogastroenterol Motil 2014;26:705-714)

\section{Ji Won Kim}

Department of Internal Medicine, Seoul National University Boramae Hospital, Seoul National University College of Medicine, Seoul, Korea

\section{Summary}

Irritable bowel syndrome (IBS) is characterized by heterogeneous pathogenesis and many IBS patients suffer from visceral hypersensitivity. Visceral hypersensitivity is evaluated under stimulated condition with mechanical distension using barostat to provide sensations of discomfort or visceral pain in most clinical researches. Distensions administered to rectum using the barostat, can induce visceral discomfort or pain, which are like typical symptoms for IBS. In addition, increased rectal sensitivity has found to be related to symptom severity in daily life. It is known that visceral hypersensitivity was associated with increased intestinal permeability and inflammation. Increased intestinal permeability leads to translocation of bacteria and their products, resulting to induce hypersensitivity by targeting the enteric neurons. Increased release of immunologic mediators resulted in visceral hypersensitivity via sensitization of neuronal cells.

Previous studies with probiotic therapeutic effects have focus on daily symptoms in IBS patients, and the effects were a small but significant improvement in overall symptom score. However, in previous studies, heterogeneous groups of IBS patients have been included rather than a selection of IBS patients based on pathophysiological characteristics. None of the studies up to now has chosen an approach by evaluating effects of probiotics targeting on one common pathophysiological mechanism. In this study, Ludidi et $\mathrm{al}^{1}$ investigated that whether implementing probiotics in IBS patients with documented visceral hypersensitivity had potential effect to reduce visceral hypersensitivity (primary end point).

This study was performed a randomized, placebo-controlled, double-blind trial in 40 Rome III IBS patients with visceral hypersensitivity. Prior to participation, patients kept a 2-weak symptom diary and underwent barostat measurement. When visceral hypersensitivity was confirmed, patients were enrolled this study and received a multispecies probiotics ( 6 different probiotic strains $10^{9} \mathrm{CFU} / \mathrm{g}$ ), or a placebo products of one sachet $(5 \mathrm{~g})$ per day for 6 weeks. At the end of the intervention period, visceroperception and symptoms were reevaluated.

In total, 35 patients were completed the study (19 in pro-

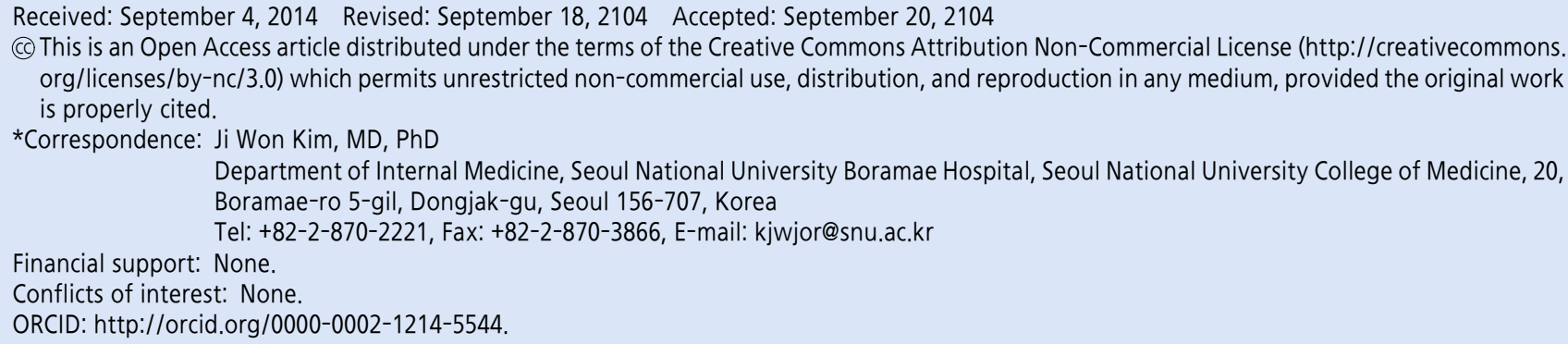


biotics and 16 in placebo groups). At baseline, all included patients showed visceral hypersensitivity, but the percentage of hypersensitive patients decreased significantly within both the probiotics and placebo groups after 6 weeks of intervention (toward $76.5 \%$ and $71.4 \%$, respectively; both $P<0.05$ vs. baseline). However, response rates did not differ between the probiotic and placebo groups $(P=0.24)$. In addition, median thresholds for pain, urge and discomfort did not differ significantly before vs. after intervention within the probiotic nor the placebo group. The difference in first sensation to rectal distension was not significant between probiotic vs. placebo group $(0.00$ [ -0.17 to 15.00$]$ vs. 0.00 [ -17.00 to 23.00$] ; P=0.78)$.

Mean symptom improvement was not significantly different between probiotic vs. placebo group ( $19 \%$ vs. $39 \%, P=0.18$ ), but interestingly there were gender-related response differences in the placebo group. In placebo group, bloating, abdominal cramping and symptom-free days only improved in the female patients, but not in the male patients. However, this gender-difference was not observed in the probiotic group.

The authors concluded that in targeted subgroups of IBS patients with a common pathophysiological characteristics (documented visceral hypersensitivity), no significant effect of a multispecies probiotic on visceroperception was observed.

\section{Comments}

IBS is a functional bowel disorder characterized by abdominal pain or discomfort relieved by defecation, and accompanied by changes in bowel habits such as diarrhea or constipation, which cannot be explained by structural, biochemical or metabolic abnormalities. The pathophysiology of IBS is known to associated with visceral hypersensitivity, altered intestinal permeability, genetic or environmental factors, psychological factors or a dysregulatd barin-gut axis. ${ }^{2}$

Visceral hypersensitivity is known to play an important role for IBS symptom, but others insist that increased sensitivity is merely a reflection of psychological factors such as anxiety or depression. Visceral hypersensitivity seemed to be important marker for differentiating IBS from functional abdominal pain syndrome (FAPS), showing hyposensitivity to physiologic distention, although IBS and FAPS had common clinical features. ${ }^{3}$ Posserud et $\mathrm{al}^{4}$ investigated the association between rectal perception and gastrointestinal (GI) and psychological symptoms in 109 IBS patients, and they showed that altered rectal perception was common in patients with IBS and was asscociated with GI symp- tom severity, especially abdominal pain and bloating. In IBS patients, rectal compliance and threshold for pain was decreased, and hypersensitivity to rectal balloon distension occurred in $33 \%$ and was associated with increased symptom severity. ${ }^{5}$

The therapeutic mechanisms of probiotics include effects on regulation of intestinal microbial homeostasis, interference with the ability of pathogens to colonize and infect the mucosa, modulation of local and systematic immune response, and stabilization or maintenance of the GI barrier function. ${ }^{6}$ Previous researches assessing the effects of probiotics on IBS symptoms have revealed conflicting data. In recent studies, it was shown that 4-week treatment with probiotics improved IBS symptoms and altered composition of the microbiota, and that probiotic treatment in IBS patients reduced the genus Bacteroids to the levels of healthy controls and also improved IBS symptoms. ${ }^{7,8}$ However, in other study Lactobacillus plantarum MF1298 species had aggravated IBS symptom score, and this means not all strains with in vitro demonstrated probiotics actually showed therapeutic effects on the in vivo condition. ${ }^{9}$

To date, clinical research with probiotics in IBS patients suffered from methodological limitations such as differences in study design, type of probiotics administered, and outcome parameters. Furthermore, previous studies frequently used subject endpoints and included heterogeneous patient populations. ${ }^{10}$ In contrasts, the most strength of this study was that the authors evaluated objective primary endpoints, which was the assessment the effect of probiotics on visceral hypersensitivity measured by rectal barostat examination. Furthermore, relatively homogenous IBS patients were specifically selected for this study based on the presence of rectal hypersensitivity using rectal barostat results. ${ }^{1}$ Interestingly in this study, the authors observed symptoms of flatulence, bloating and abdominal cramping improved in placebo group, but not in probiotic group, and they assumed that the presence of some strains in the probiotic mixture may have contributed to an increased gas production and thereby to an increase in gas-related symptoms.

There were several limitations in this study. First the duration of probiotic supplementation period was 6 weeks. As IBS is a chronic condition, it may require longer duration to result in significant effects at symptom level. Secondly, a mixture of 6 different bacterial strains used in this study, and the bacterial competitive interaction might occur resulting in negative effect on outcome. Lactobacillus acidophilus NCFM was used based on its antinociceptive properties, and Lactobacillus salivarius and Lactobacillus casei were selected by their in vivo potential to im- 
prove epithelial integrity and inflammatory status. ${ }^{11}$ Thirdly, in contrast to conventional chemistry-based drugs which act after absorption in the small bowel, probiotics need to reach a subject's intestine alive to show the effect. Therefore, it is important to confirm alive multispecies probiotics in the feces of patients.

In conclusion, the multispecies probiotics has no effect on visceroperception in IBS patients with visceral hypersensitivity in placebo-controlled study, there are possibility of other mechanism of multispecies probiotics may contribute to the relieving IBS symptoms. Further researches should be performed to identify which strains of probiotics provide the optimal therapeutic effect to individual patients with IBS and which specific symptoms of IBS should be the aims of probiotic treatment.

\section{References}

1. Ludidi S, Jonkers DM, Koning CJ, et al. Randomized clinical trial on the effect of a multispecies probiotic on visceroperception in hypersensitive IBS patients. Neurogastroenterol Motil 2014;26:705714 .

2. Lee KN, Lee OY. Intestinal microbiota in pathophysiology and management of irritable bowel syndrome. World J Gastroenterol 2014;21:8886-8897.

3. Nozu T, Okumura T. Visceral sensation and irritable bowel syndrome; with special reference to comparison with functional abdominal pain syndrome. J Gastroenterol Hepatol 2011;26(suppl 3):122-
127.

4. Posserud I, Syrous A, Lindström L, Tack J, Abrahamsson H, Simrén M. Altered rectal perception in irritable bowel syndrome is associated with symptom severity. Gastroenterology 2007:133:11131123.

5. Van der Veek PP, Van Rood YR, Mascles AA. Symptom severity but not psychopathology predicts visceral hypersensitivity in irritable bowel syndrome. Clin Gastroenterol Hepatol 2008;6:321-328.

6. Cha BK, Jung SM, Choi CH, et al. The effect of a multispecies probiotic mixture on the symptoms and fecal mirobiota in diarrhea-dominant irritable bowel syndrome. A randomized, double-blind, placebo-controlled trial. J Clin Gastroenterol 2012;46:220-227.

7. Yoon JS, Sohn W, Lee OY, et al. Effect of multispecies probiotics on irritable bowel syndrome: a randomized, double-blind, placebo-controlled trial. J Gastroenterol Hepatol 2014;29:52-59.

8. $\mathrm{Ng} \mathrm{SC,} \mathrm{Lam} \mathrm{EF,} \mathrm{Lam} \mathrm{TT,} \mathrm{et} \mathrm{al.} \mathrm{Effect} \mathrm{of} \mathrm{probiotic} \mathrm{bacteria} \mathrm{on} \mathrm{the}$ intestinal microbiota in irritable bowel syndrome. J Gastroenterol Hepatol 2013;28:1624-1631.

9. Ligaarden SC, Axelsson L, Naterstad K, Lydersen S, Farup PG. A candidate probiotic with unfavourable effects in subjects with irritable bowel syndrome: a randomized controlled trial. BMC Gastroenterol 2010;10:16.

10. Sisson G, Ayis S, Sherwood RA, Bjarnason I. Randomized clinical trial: a liquid multi-strain probiotic vs. placebo in the irritable bowel syndrome - a 12 week double blind study. Aliment Pharmacol Ther 2014;40:51-62.

11. Timmerman HM, Niers LE, Ridwan BU, et al. Design of a multispecies probiotic mixture to prevent infectious complications in critically ill patients. Clin Nutr 2007;26:450-459. 